\title{
One Case of Pelger's Familiar Nuclear Anomaly of Leucocytes (the First Case Reported in Japan); Recommendation of Two \\ Expressions: "Pseudo-myelocyte" (Undritz) and \\ "Pseudo-metamyelocyte" and Suggestion of "Half Typical Pelger's Anomaly."
}

5th Hematological Paper.

By

Yutaka Kokubo.

(小久保 裕)

(From the Pediatric Department, Faculty of Medicine Tohoku Imperial University, Sendai. Director: Prof. A. Sato.)

\section{Introduction.}

Two patients with a new blood picture were found by Pelger ${ }^{13}$ in Amsterdam in 1930, who had investigated their nuclear shift. In these cases, the nuclear shift to the left was so much pronounced, that there was no cells, or almost none of Class III. In 1932, two years later than his publication, $\mathrm{Hu} \mathrm{et}^{22}$ reported two families in Holland with this nuclear anomaly, though one of the very first cases of Pelge $r$ belonged to one of these two families. Then Herman $\nabla o s^{33}$ reported of another family with the anomaly in Holland. In $1933 \mathrm{Schilling} \mathrm{g}^{4)}$ suggested the name of Pelger's Familiar Nuclear Anomaly (Pseudokernvershiebung) of leucocytes to this blood picture with the peculiar nuclear anomaly. Then Burge $\mathrm{r}^{5)}$ published two further families in Holland, Weigerd $t^{6)}$ one family in Germany, Undritz $z^{\eta)}$ one family

1) Pelger, cited from $H$ u ët's report.

2) G. J. H u ët, Kl. Wschr., 1932, 1264.

3) Herman $\nabla$ os, eited from $H u$ ë t's report.

4) V. Schilling, Dtsch. med. Wschr., 1933, 59, 724.

5) Burger, cited from $S c h i l l i n g ' s$ report.

6) Weigerdt, cited from S chilling's report.

7) E. Undritz, Schweiz. med. Wschr., 1933, 286; 1934, 10. 
in Switzerland, Schilling* one family in Checho-Slovakia, Zü u de ${ }^{8)}$ another family in Germany, and Chevallier and Ely** one case in France.

In our country Pelger's anomaly has not yet been reported thus far. In my daily examination of blood pictures of lactating mothers with positive and negative Arakawa's reaction in our Pediatric Department, I have come across a case of the anomaly and desire to report of the case and of her family as well as of some of her relatives.

\section{My Own Cases.}

The details of my own cases will be seen from the accompanying tables (Cf. Table I and II and Fig. 1). In Tables I and II the word "peculiar" means "peculiar form of nucleus" (Cf. Fig. 2 and 3). Namely, nuclei of cells of Class I show, as will be seen from Fig. 3, very peculiar forms; they are round, kidney-shaped, sausage-or pea-

\section{TABLE I.}

Blood picture of Yamag.....Yamas..... Families with Pelger's Familiar Nuclear Anomaly (Part I).

\begin{tabular}{|c|c|c|c|c|c|c|c|}
\hline Blood relation & \multicolumn{2}{|c|}{ Mother } & Son & $\underset{\text { ter }}{\text { Daugh- }}$ & Son & $\begin{array}{c}\text { Daugh- } \\
\text { ter }\end{array}$ & Son \\
\hline Age $\left\{\begin{array}{l}\mathbf{Y}=\text { yearg } \\
\mathbf{M}=\text { months }\end{array}\right.$ & \multicolumn{2}{|c|}{$39 \mathrm{y}}$. & $13 \mathrm{y} .5 \mathrm{~m}$ & $11 \mathrm{y}$. & $8 \mathrm{y} .7 \mathrm{~m}$. & $5 \mathrm{y} .9 \mathrm{~m}$. & $3 \mathrm{~m}$. \\
\hline $\begin{array}{l}\text { Hemoglobin (Sahli } \\
\text { value) }\end{array}$ & & 100 & 100 & 90 & 80 & 90 & \\
\hline $\begin{array}{l}\text { cmm. (thousand) } \\
\text { Reticulocyte (\%) }\end{array}$ & 5,300 & $\underset{\mathbf{2}}{5,290}$ & 4,850 & $\begin{array}{l}5,280 \\
3.5\end{array}$ & 5,440 & $\begin{array}{c}5,140 \\
5\end{array}$ & 5,600 \\
\hline $\begin{array}{l}\text { Platelets per cmm. } \\
\text { (thousand) }\end{array}$ & 280 & 360 & 300 & 310 & 320 & 390 & 321 \\
\hline 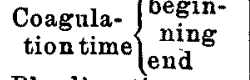 & & & & $\begin{array}{l}\mathbf{3}^{\prime} \\
\mathbf{5}^{\prime}\end{array}$ & $\begin{array}{l}2 / 30 \prime \prime \\
5^{\prime}\end{array}$ & $\begin{array}{l}3^{\prime} \\
6^{\prime}\end{array}$ & \\
\hline $\begin{array}{l}\text { Bleeding time } \\
\text { Blood group } \\
\text { White blood cells }\end{array}$ & 0 & $\begin{array}{l}3^{\prime} \\
0\end{array}$ & $\begin{array}{c}2 / 30 \prime \prime \\
A\end{array}$ & $\begin{array}{c}2 / 30 \prime \prime \\
\mathrm{A}\end{array}$ & $\mathrm{A}^{2 \prime}$ & $\mathrm{A}^{2 \prime}$ & $\begin{array}{c}2^{\prime} \mathbf{4} 0^{\prime \prime} \\
\mathrm{A}\end{array}$ \\
\hline & 5,800 & 7,800 & 9,200 & 8,100 & 8,800 & 9,200 & 10,700 \\
\hline
\end{tabular}

* In fact, Schilling examined the blood specimens sent to him which a doctor obtained from a case in Checho-Slovakia.

8) W. Z ii nde 1, Fol. Haematol., 1935, 54, 1.

* After I read the paper at the 96 monthly meeting of the Tohoku Medical Society, held in Sendai, May 15th, 1936, I saw that a case was a reported by Chevallier and Ely in the Sociéte Francase D'Hématologie, February 5th 1936, but the details have not yet been published (Cf. M.P. Chevallier and M.Z. E 1 y, La presse médicale, 1936, No. 20, 386). 


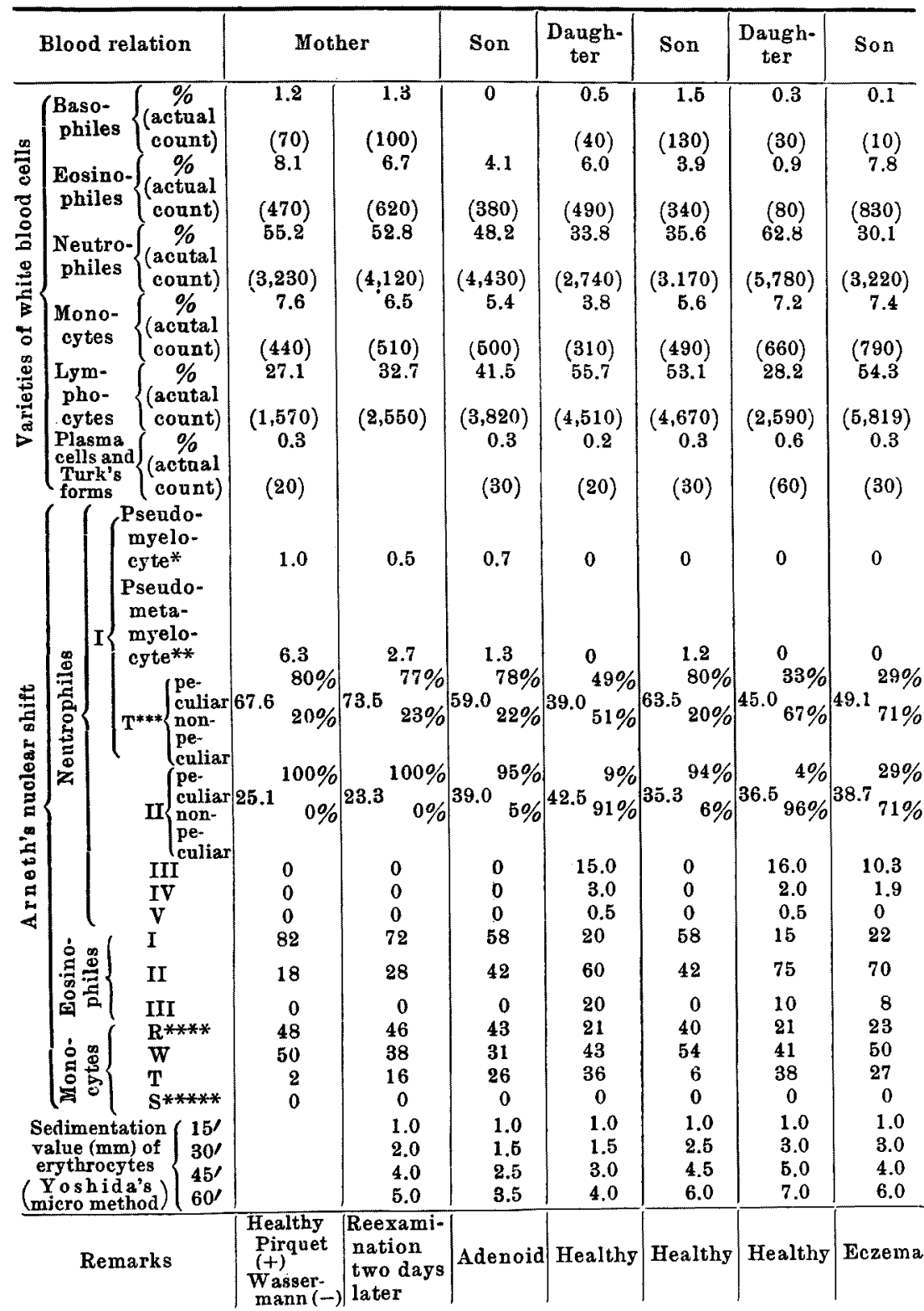
Cf. Text as to their real nature

*** T......metamyelocytes with deeply indented nucleus

**** $\mathbf{R}$......cells with round-formed nucleas

$* * * * *$ S ......cells with polymorph or segmented nucleus 
TABLE II.

Blood picture of Yamag.....Yamas..... Families with Pelger's Familiar Nuclear Anomaly (Part II).

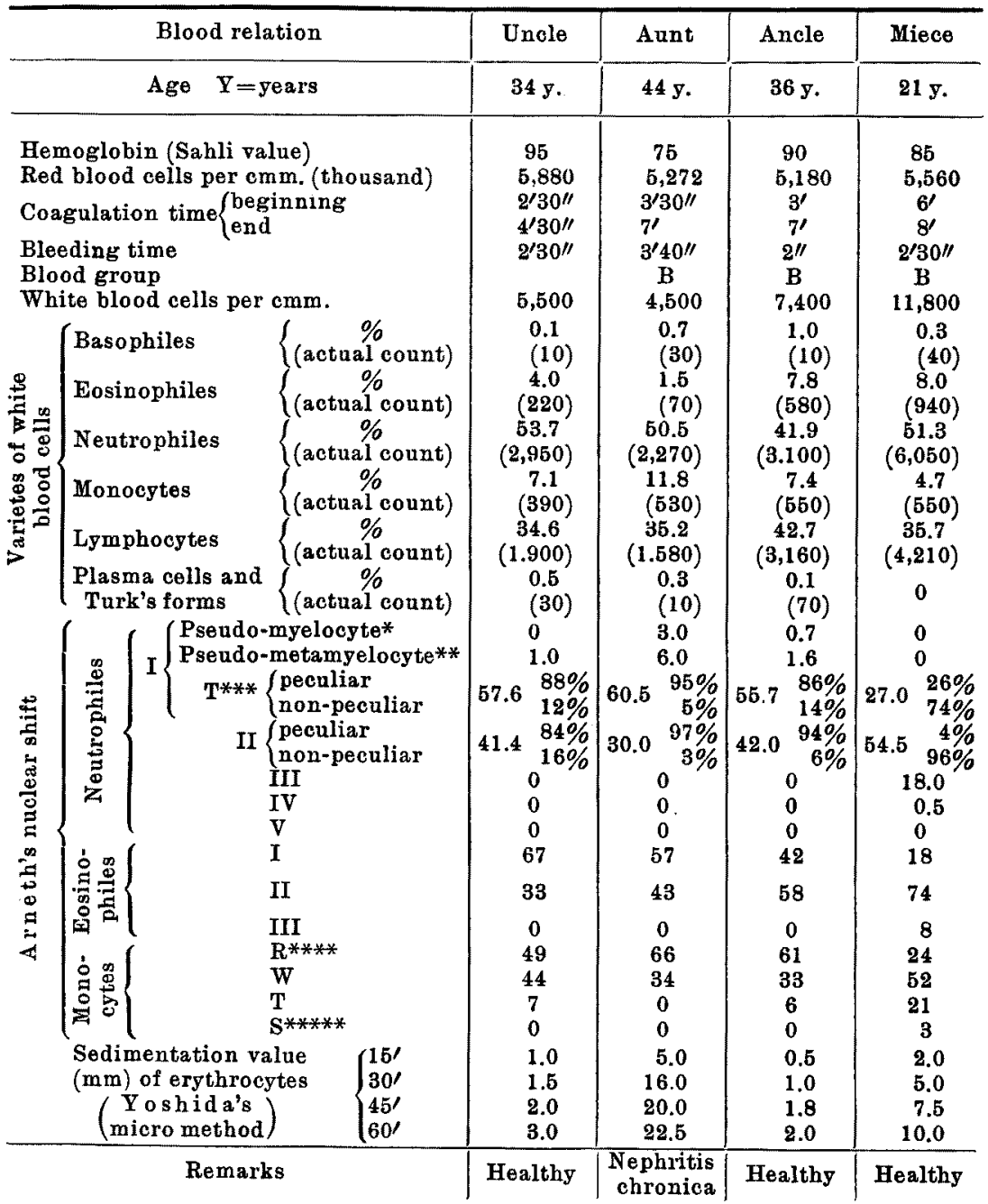

$*, * *, * * *, * * * *, * * * * *$ Cf. the foot note of Table I.

nut-shaped, or of the form of kidney with a rather deep or very deep indentation, or of a dumb-bell. Neutrophile cells with such nuclei(Cf. $M$ and $W$ under I, Tables I and II) might be considered in a usual hemogram (of Arneth) as representing myelocytes (M), metamyelocytes 
Fig. 1. Family tree of Yamag...... Yamas...... Families with Familiar Nuclear Anomaly.
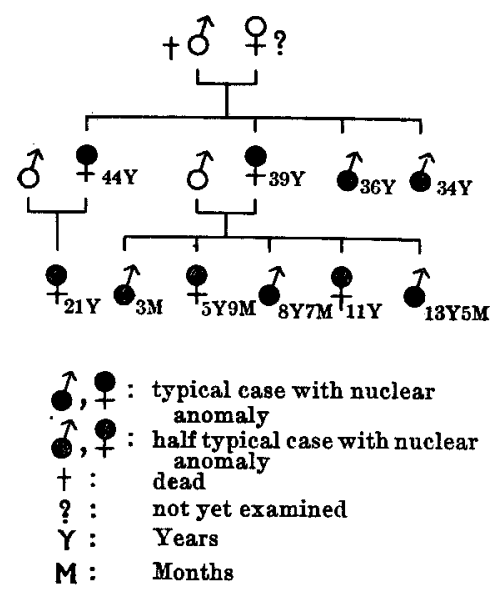

with slightly indented nucleus (W), and metamyelocytes with deeply indented nucleus (T) respectively, but in the present table they are almost all of them not young forms but mature cells. This is a very interesting point; any laborant with an experience of the Tohoku-Pediatric Method ${ }^{9}$ of the peroxidase stain will recognize the difference due to two features. One of these is the peroxidase granules of these cells in the present cases; they are just such granules as would be seen in polymorphonuclear neutrophiles, quite different from those thick granules seen in myelocytes or in meta-

myelocytes with slightly indented nucleus. This feature is especially important, because any difference of cytoplasma has never been noticed in the hitherto published reports on Pelger's anomaly.

The second feature is that of their nuclei. These are stained as deeply as those of polymorphonuclear neutrophiles and show distinctly safranin-stained chromatin-net, while nuclei of myelocytes and metamyelocytes with slightly indented nucleus with fine chromatinnetwork are stained only lightly red with safranin. This second feature was already noticed by Undritz in 1933 in the Gie m sa-stained films; he described full details of the nuclear peculiarities of these cells. This second feature was also mentioned by Zündel in 1935, though he used the Giems a stain probably.

These two features-one described by my myself and the other by Undritz and $Z$ ündel-are very important and suggest a further merit of the copper peroxidase method, because with this method a hematologist will be able to recognize very easily such a "Pseudomyelocyte" (Und ritz) or " Pseudo-metamyelocyte" in the very first microscopic field with such a peculiar mononuclear cell. "Pseudomyelocyte" or "Pseudo-metamyelocyte" may thus well be called a mature neutrophile cell with a mature, only not-segmented nucleus.

9) A. Sato, T. Suzuki and R. Shibata, Tohoku J. Exp. Med., 1934, 24, 195. 
Thus the anomly of the family may be defined as consisting in a primitive and incomplete differentiation and a strange form of the mature nucleus of mature neutrophile leucocytes.

Neutrophiles of Class II are also somewhat characteristic, because the two segmented nucleus in those cells is different from that in the usual cells of Class II; each segment is quite round and connected to the other with a very fine, yet very distinctly stained filament (Cf. Figs. 2 and 3). This characteristic was, though noticed by all the authors, emphasized by $\mathrm{Zündel}$.

As has been shown in Tables I and $\Pi$, six persons, that is, mother, first child (male), third child (male) two uncles and one aunt showed a typical Pelger's anomaly. As Pelger and the other authors stated in their papers, each case showed apparently younger forms of neutrophiles exclusively, while no polynuclear cells were seen in each of my own cases. It is interesting that apparent myelocytes (really "Pseudo-myelocyte") and apparent metamyelocytes (really "Pseudo-metamyelocyte") occurred in these cases in spite of the fact that all of them were healthy with the exception of the aunt suffering from chronic nephritis. And it is still more interesting that in this nephritic aunt "Pseudo-myelocytes" and "Pseudo-metamyelocytes" occurred in a far larger number than in the five healthy cases. It will be no wonder that when a healthy person with Pelger's anomaly happens to be taken ill, the nuclear shift to the left may be found very severe. In the typical cases of my own, cells over and of Class III were not seen, and the percentage of the peculiar forms in metamyelocytes with deeply indented nucleus and cells with Class II is from $77 \%$ to $100 \%$. In the other four cases something which has never been noticed before will be described below:-

In these four cases, the degree of the nuclear shift to the left is far weaker than in the typical cases, though the shift in those cases themselves is different from that to be seen in a usual healthy person. Indeed the nuclear peculiarity in these four non-typical cases is not so pronounced as in the typical cases, but nevertheless their nucleus show Pelger's anomaly in a modified form. These four non-typical persons show a very severe nuclear shift to the left in spite of their being in excellent health, except the 3rd son suffering from eczema, and in spite of an otherwise normal blood picture. Besides, cells with peculiar forms of nucleus which show an inclination to the typical nuclear form seen in Pelger's anomaly are of from $39 \%$ to $26 \%$ in the Class of metamyelocyte with deeply indented nucleus, and of from 
$29 \%$ to $4 \%$ in Class II. From this fact I should like to believe that these four cases belong to Pelger's anomaly, and I desire to give to these cases "Half Typical Pelger's anomaly."

As to eosinophile and basophile leucocytes, and as to monocytes as well as neutrophiles, all the authors state that the apparent or pseudo nuclear shift to the left is seen in each cell group just mentioned. In my own cases eosinophiles and monocytes showed also the pseudo shift, but as to basophiles I shall be able to relate of the result in the near future. The pseudo shift of eosinophiles and monocytes in also seen in the half-typical cases as well as in the typical cases of my own, though in the former the shift was not so pronounced as in the latter. Heredity has been shown in all the hitherto published reports. In my own case a heredity will be seen as is shown in Fig. 1 (Cf. Family Tree of Yamag......Y $a$ mas...... Families with Familiar Nuclear Anomaly).

To the present family I have given the name of "Yamag...... Yamas..... Families." Yamag...... is the maternal family name and Yamas......the paternal. As to the blood investigation of the maternal parents, their brothers and sisters, I shall be able to report in the near future.

\section{Remarks.}

By way of appendix, I desire to call the special attention of readers to the following points:-

1. "Pseudo-myelocyte"-a neutrophile mononuclear leucocyte, resembling a myelocyte or an apparent myelocyte with mature nucleus and mature cytoplasma. Though the characteristic feature of the nucleus will, as stated above, be recognized by use of the Giemsa stain, the cytoplasma as well as the nucleus will be seen very easily by use of the Tohoku-Pediatric Method, a modification of Sato and Sekiya' $\mathbf{s}^{10)}$ copper peroxidase reaction.

2. "Pseudo-metamyelocyte"***_an apparent metamyelocyte with slightly indented nucleus, a neutrophile leucocyte with mature nucleus and with cytoplasma. Both these will be recognized very easily by use of the Tohoku-Pediatric Method, though the feature of the nucleus can also be shown by the Giems a stain.

10) A. S a to and S. Sekiy a, Tohoka Igaku-Zasshi, 1920, 6, 534; Tohoku J. Exp. Med., 1926, 7, 111.

*** I wish to use the "Pseudo-metamyelocyte" instead of the word "Pseudojugendliche" neutrophile leucocyte (Undritz). 
3. "Pseudo left shift"-an apparent shift of nuclei of neutrophiles to the left due to the prevalence of "Pseudo-myelocyte" and "Pseudo-metamyelocyte". This has already been expressed in the word "Pseudokernvershiebung" - "Pseudo nuclear shift"-by Schilling."1)

4. Suggestion of the expression "Half Typical Pelger's Anomaly". Here I desire to suggest a new expression of "Half Typical Pelger's Anomaly" for the state which has never been noticed before and which is important for the prevention of a possible diagnostic error. A person with the "Half Typical Pelger's Anomaly" may be diagnosticated as suffering from a disease accompanied by a left shift in spite of the fact that all the blood picture is entirely normal. As this picture is not so extreme as in Pelger's Anomaly, such a diagnostic error may easily happen, without leading to a search of blood anomaly of his family. A hematologist or an examiner of nuclear shift should bear in mind that there is a healthy person with "Half Typical Pelger's Anomaly" as well as a healthy one with Pelger's Anomaly.

\section{Conclusions.}

In the present paper I demonstrate the Yam ag......Y a mas...... Families as one family of Pelger's familiar nuclear anomaly of leucocytes in this country. And this is the first case ever reported in Japan.

In the investigation of the anomaly, the use of the ToholsuPediatric Method, a modification of Sato-Sekiya's Copper peroxidase Reaction, has shown an advantage over the Giemsa stain, because that method show "Pseudo-myelocyte" (Undritz) and "Pseudo-metamyelocyte" quite distinctly, so that the "Pseudo left shift" can be differentiated very easily from the real left shift.

In this paper I have described four cases of the "Half Typical Pelger's Anomaly" which has never been noticed before.

I should like to suggest giving to the abnormal leucocytes described in the present paper the following names: "Pseudo-myelocyte" (Undritz) and "Pseudo-metamyelocyte" (Undritz-Kokubo).

Prof. A. Sato.

11) V.Sehilling, Das Blutbild und seine klinische Verwertung, Jena 1933, 181. 